\title{
Assessing operational complexity of manufacturing systems based on algorithmic complexity of key performance indicator time-series
}

\author{
Bugra Alkan ${ }^{\mathrm{a}}$ and Seth Bullock ${ }^{\mathrm{a}}$ \\ aDepartment of Computer Science, University of Bristol, Merchant Venturers' Building, \\ Woodland Road, Bristol, BS8 1UB, United Kingdom
}

\section{ARTICLE HISTORY}

Compiled January 12, 2021

\begin{abstract}
This article presents an approach to the assessment of operational manufacturing systems complexity based on the irregularities hidden in manufacturing key performance indicator time-series by employing three complementary algorithmic complexity measures: Kolmogorov complexity, Kolmogorov complexity spectrum's highest value and overall Kolmogorov complexity. A series of computer simulations derived from discrete manufacturing systems are used to investigate the measures' potentiality. The results showed that the presented measures can be used in quantitatively identifying operational system complexity, thereby supporting operational shop-floor decision-making activities.
\end{abstract}

\section{KEYWORDS}

Manufacturing systems; Complexity; KPI; Kolmogorov complexity; Operations research; Discrete event simulations

\section{Introduction}

Manufacturing companies have to cope with an uncertain and volatile environment driven by factors such as rapidly changing customer demands, political regulations, technological advancements and global market competition to remain profitable and competitive (H. ElMaraghy et al., 2013). The internal complexity of a manufacturing company is linked to these factors, where they manifest themselves as an increased number of product variants, high product complexity, a high number of diverse customers, and increased number and variety of business targets (Alkan, 2019). This ultimately results in an increase in operational uncertainty which may result in unpredicted/unexpected manufacturing system behaviours (Alkan, Vera, Ahmad, Ahmad, \& Harrison, 2018; Irani, 2010).

An increase in complexity may decrease the responsiveness of manufacturing systems and make them harder to manage and control (Alkan, 2018). As an example, increasing product variety can tend to encourage manufacturing systems to have a higher degree of flexibility for handling multiple components due to the increased variety of product parts. This often results in complex and sophisticated system structures where a high number of mechatronic components and software algorithms need to cooperate to achieve a set of pre-defined production goals. Without proper IT sys-

Contact: Bugra Alkan, e-mail: B.Alkan@bristol.ac.uk, Address: Department of Computer Science, University of Bristol, Merchant Venturers' Building, Woodland Road, Bristol, BS8 1UB, United Kingdom 
tems and complexity management strategies, an increase in system complexity may decrease the operational efficiency of the entire facility, and result in line-balancing problems especially during disruptive events where system managers are required to make correct decisions on time.

One effective way to design manufacturing systems that are diagnosable, predictable and productive is the systematic assessment of complexity, allowing us to identify excessive/harmful complexity, and hence to take steps to reduce it and/or manage its implications. Analysis and quantification of complexity also allow us to develop and implement the correct strategies required for its management (Efthymiou, Mourtzis, Pagoropoulos, Papakostas, \& Chryssolouris, 2016). The literature reveals two types of manufacturing systems complexity, i.e. structural (static) and operational (dynamic) (Frizelle \& Woodcock, 1995). Structural complexity is linked to the time-independent characteristics of a manufacturing system and relates to the types and variety of subsystems and their interactions (Deshmukh, Talavage, \& Barash, 1998). Operational complexity, on the other hand, is induced by systems' time-dependent characteristics and involves the aspects of flows, lags and stochasticity (Frizelle \& Suhov, 2001). Please note that there is a close bi-directional relationship between the structural and operational complexity of manufacturing systems (Alkan, 2018).

In the manufacturing context, complexity is often defined as the uncertainty associated with the information required to describe the overall state of a manufacturing system and/or its components (Deshmukh et al., 1998). In this context, uncertainty is measured by Shannon metric based on Boltzmann's entropy (Shannon, 2001) which is the average rate at which information is generated by a stochastic source of data. In the literature, there are many studies employing Shannon entropy to measure structural (Deshmukh et al., 1998; Efstathiou, Calinescu, \& Blackburn, 2002; Frizelle \& Woodcock, 1995; Kohr et al., 2018; Z. Zhang, 2012) and operational complexity (Calinescu, Efstathiou, Schirn, \& Bermejo, 1998; Chryssolouris, Efthymiou, Papakostas, Mourtzis, \& Pagoropoulos, 2013; Efthymiou, Pagoropoulos, Papakostas, Mourtzis, \& Chryssolouris, 2014; Frizelle \& Suhov, 2001; Kohr et al., 2018; Mourtzis, Doukas, \& Psarommatis, 2013; Sivadasan, Smart, Huaccho Huatuco, \& Calinescu, 2010; Vrabič \& Butala, 2011; Y. Wu, Frizelle, \& Efstathiou, 2007; Y. R. Wu, Huatuco, Frizelle, \& Smart, 2013; T. Zhang \& Efstathiou, 2006). Although, entropic measures provide an objective way for quantifying complexity, they are criticised for involving subjectivity in defining the resource states (e.g. busy, idle, etc.) (Papakostas, Efthymiou, Mourtzis, \& Chryssolouris, 2009), and being tied to the level of detail (Sivadasan, Efstathiou, Calinescu, \& Huatuco, 2006).

Another quantitative approach towards the definition of manufacturing systems complexity is based on chaos and non-linear dynamics theory. Examples include (Chryssolouris, Giannelos, Papakostas, \& Mourtzis, 2004; Donner, Scholz-Reiter, \& Hinrichs, 2008; Giannelos, Papakostas, Mourtzis, \& Chryssolouris, 2007; Katzorke \& Pikovsky, 2000; Massotte, 1996; Papakostas \& Mourtzis, 2007; Schmitz, Van Beek, \& Rooda, 2002; Scholz-Reiter, Freitag, \& Schmieder, 2002; Wiendahl \& Scheffczyk, 1999). These methods include phase space reconstruction techniques, maximal Lyapunov exponent testing, and the use of bifurcation diagrams (Alkan \& Harrison, 2019). Nevertheless, these approaches can be considered as limited, since they are unable to capture the effects of stochastic events such as machine breakdowns (Efthymiou, 2013), and (with the exception of Lyapunov exponent testing methodology) are tied to the schematic analysis of dynamic behaviours (Efthymiou, Pagoropoulos, Papakostas, Mourtzis, \& Chryssolouris, 2012). According to Efthymiou (2013), these approaches also require relatively large data sets and are highly sensitive to disturbances in mea- 
surement.

Manufacturing systems complexity can also be assessed through qualitative and hybrid measures. Hybrid measures merge information theory and survey-based assessments and are often employed to provide an industrially readable picture of complexity. Example studies include (Ahmad et al., 2016; Alkan, Vera, Ahmad, Ahmad, \& Harrison, 2016b; Alkan, Vera, Chinnathai, \& Harrison, 2017; H. A. ElMaraghy, 2005; W. ElMaraghy \& Urbanic, 2003; Garbie \& Shikdar, 2010; Kim, 1999; S. Samy \& ElMaraghy, 2012; Sarkis, 1997; Schoettl, Paefgen, \& Lindemann, 2014; Windt, Philipp, \& Böse, 2008). Hybrid measures are often considered advantageous as they are easy to apply in real systems and considered an effective approach in comparing system alternatives during design stages (Alkan, Vera, Ahmad, Ahmad, \& Harrison, 2016a). According to Alkan et al. (2018), these measures are limited in the sense that they are often designed for a specific purpose or application. Moreover, they are incapable of capturing intricate structural patterns, and therefore lack the deeper insight into manufacturing systems complexity that more quantitative measure promise.

In addition to its objectivity, complexity has also a subjective nature; being dependent on the context and observer (Gell-Mann, 1995). This type of complexity is termed as "perceived complexity" and often assessed using structured or semi-structured surveys and questionnaires (Calinescu et al., 1998; Falck, Örtengren, \& Rosenqvist, 2012; Kohr et al., 2018; Mattsson, Gullander, \& Davidsson, 2011; Mattsson, Tarrar, \& FastBerglund, 2016). Although these approaches can capture the perceived level of complexity and highlight problems in existing systems, they are incapable of evaluating/comparing alternative systems in early design stages since no physical mock-up or process trials are available (Alkan et al., 2018). Also, they are limited to survey stages, and their results are dependent on the subjective interpretation of the interviewees (Alkan, Vera, Ahmad, \& Harrison, 2017).

Although the existing approaches have resulted in valuable results, only a few of them (Chryssolouris et al., 2013; Efthymiou et al., 2014; Schmitz et al., 2002; Vrabič \& Butala, 2011) have attempted to investigate the relationship between complexity and manufacturing key performance indicators (KPIs). Henceforth, this article aims to contribute to a better understanding of the above-mentioned link between complexity and manufacturing systems' KPIs through the application of three complementary Kolmogorov complexity measures. Towards this aim, a data-driven operational manufacturing systems complexity quantification approach is proposed and illustrated on two discrete production system simulation models. The proposed approach includes the first-time implementations of both Overall Kolmogorov complexity (KLO) and Kolmogorov complexity spectrum maximum value (KLM) measures in the domain of manufacturing, and quantitatively links operational complexity to manufacturing KPIs; thereby supporting operational shop-floor decision-making activities in an explicit way.

The rest of the paper is organised as follows. Section 2 reports the research background, i.e. the terminology, manufacturing KPIs and Kolmogorov complexity measures used within this research. Section 3 presents the research methodology. Section 4 addresses the case studies investigated in this research, and discusses the obtained results. In Section 5, the validity of the approach is discussed. Finally, Section 6 concludes the paper and outlines future work. 


\section{Research background}

This section provides background to the topics discussed throughout the article.

\subsection{Manufacturing KPIs}

KPIs are measurable metrics that show how successfully a company meets its key business goals. In general, manufacturing KPIs can be grouped into five main categories: cost, quality, flexibility, sustainability and time (Chryssolouris, 2013). These categories can be extended to a set of sub-categories including: availability, utilization, throughput, rework ratio, scrap ratio, machine flexibility, customer satisfaction, cycle time, flow time, corrective maintenance ratio, first time pass yield, mean time to failure, mean time to repair, overall equipment effectiveness, production effectiveness, production process ratio, quality, etc. In a manufacturing enterprise, KPIs can be tracked and monitored at various distinct levels, including machine, workstation, production line, enterprise, etc. KPIs are mainly displayed to shop-floor staff, managers and supervisors in order to support their decision-making activities (Amrina \& Vilsi, 2015). The frequency at which KPIs are monitored and assessed is vital, and mainly depends on the nature of the manufacturing operation. In general, KPIs are tracked in real-time, however, they can be displayed periodically or on-demand (Assad et al., 2019).

\subsection{Time-series}

The application of the Kolmogorov complexity measures necessitates the consideration of manufacturing KPIs in a time-series format. A time series is a time-stamped chronological sequence of observations on a variable of interest (Montgomery, Jennings, \& Kulahci, 2015). Time series can usually be measured non-uniformly over time (i.e. discrete-time data), and hence can be represented with a time stamp vector $t_{i}$ and corresponding measurements $x_{i}$. However, they can also be uniformly sampled at a constant sampling period $\Delta t$. The analysis of time-series can be achieved through two successive steps (Deb, Zhang, Yang, Lee, \& Shah, 2017). The first step covers the obtainment of the structure and underlying pattern of the data, whereas the second step addresses the preparation of the statistical models to make future predictions. Analysis of the time series can be used for many purposes including economic forecasting, operation and quality control, evaluation of censuses, etc. The decomposition of the sequence into three elements, i.e. pattern, seasonality and residual, is a standard approach (Brockwell \& Davis, 2016). Trend is a pattern of continuous change or general inclination of a set of data points over time along any axis on a graph. Seasonality is the occurrence of fluctuations at specific and regular intervals, such as fluctuations across weekly, monthly, or quarterly periods. A residual is the vertical difference between a regression line and a data point. Analysis of the time-series will typically be split into univariate and multivariate analyses. Time-series consisting of single observations recorded sequentially over equivalent spans of time are known as univariate time-series. Multivariate time-series, on the other hand, involve many time series that interact simultaneously with dependent data. Examples of multivariate time-series include measuring behavioural patterns in various brain regions over time or measuring atmospheric temperature, air pressure and humidity over time, etc. 


\subsection{Kolmogorov complexity}

Kolmogorov complexity is an algorithmic complexity measure representing the degree of uncertainty in a binary time-series, and named after Andrey Kolmogorov who chiefly proposed this subject in 1963. According to Cover \& Thomas (2012), Kolmogorov complexity represents "complexity of any binary finite time-series is linked to the length of the shortest binary computer program that can reproduce this string on the Universal Turing Machine $(U)$ and then halt". Although Kolmogorov complexity cannot be directly measured, Lempel \& Ziv (1976) proposed a data compression algorithm based on the Kolmogorov's idea, which is used in measuring randomness in finite-time-series. Lempel-Ziv's approach has been used in several disciplines, including, biomedical engineering (Ibáñez-Molina, Iglesias-Parro, Soriano, \& Aznarte, 2015; Rivolta, Migliorini, Aktaruzzaman, Sassi, \& Bianchi, 2014; Y. Zhang, Wei, Liu, Zhao, \& Liu, 2016) and environmental science (Mihailović, Mimić, Drešković, \& Arsenić, 2015; Mihailović, Mimić, Nikolić-Djorić, \& Arsenić, 2015). The following section provides three complementary algorithmic complexity metrics aiming to measure Kolmogorov complexity based on the Lempel-Ziv data compression algorithm.

\section{Research methodology}

In the study presented here, operational complexity of a manufacturing system is defined as the degree of irregularity arising in its KPI time-series. In this definition, it is assumed that an increase in complexity is accompanied by an increase in the difficulty in predicting and controlling operational system efficiency. Towards this, operational complexity is assessed through three complementary Kolmogorov complexity measures, i.e. Kolmogorov complexity spectrum, Kolmogorov spectrum highest value and the overall Kolmogorov complexity. The presented method consists of three distinct steps: $i$ ) obtaining a KPI time-series either by an on-site measurement process or a discrete-event simulation (DES) model, ii) transforming the KPI time series into a Kolmogorov complexity spectrum, and iii) calculating the complexity of the timeseries based on the overall Kolmogorov complexity measure. Figure 1 depicts an overview of the methodology.

\subsection{Data preparation}

One of the key goals of non-linear time series analysis is to assess complexity which is hidden in the dynamics of the system. In the proposed approach, relevant history for the system's KPI time-series $\left(x_{i}\right)$ is collected through the periodical on-site measurements. In today's manufacturing settings, real-time production data can be collected through Internet-of-Things-enabled (IoT-enabled) field devices and stored within time-series databases such as InfluxDB (Shahid, 2019) and Prometheus (Volz \& Rabenstein, 2015). Hence, the approach can be easily embraced by Industry 4.0 aligned data-analytics and visualisation systems in which KPI time-series are used to enhanced flexibility in decision-making and production forecasting. In addition to this, KPI time-series data can be gathered/analysed via discrete event simulation (DES) models in the case where the physical system mock-up is not available. DES models can be used in optimising various system design and operational parameters, such as the configuration of resources, processing times, buffer capacities, set-up time of machines, etc., and hence can be an effective tool in measuring operational complexity of 


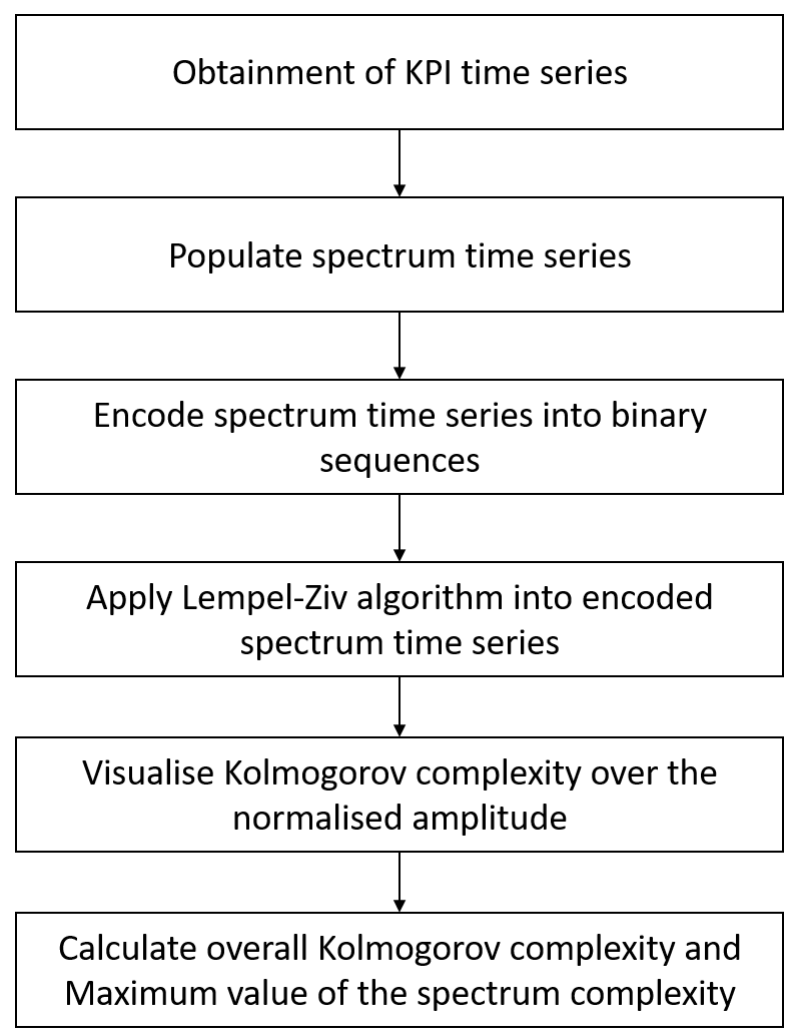

Figure 1. Overview of the methodology.

manufacturing systems with respect to given operational conditions and parameters. Please note that, as Kolmogorov complexity measures are very sensitive to the length of time series, the observation period and sample size are essential validation criteria for both on-site measurements and DES models in the proposed method. According to Yentes et al. (2013), measurements of algorithmic complexity measures are especially sensitive to very small data sets, thus, they suggest calculating measures over a sample of at least 200 observation points. Hence, we will consider 200 as a minimum sample size for KPI time series observed over equal time intervals. However, as part of future work described at the end of this paper, there are plans to perform more sensitivity analysis for the presented measures.

\subsection{Measuring Kolmogorov complexity}

Once the selected KPI time-series is attained, its Kolmogorov complexity over a range of amplitude can be investigated. The steps of the calculation of Kolmogorov complexity of a finite time-series $\left(x_{i}\right) i=1,2,3 \ldots, N$ by the Lempel and Ziv compression algorithm (LZA) are given as follows.

- Encode the time-series by creating a binary sequence consisting of the characters 0 and 1 according to the rule described below. 


$$
S(i)= \begin{cases}0 & \text { if } x_{i}<x_{t} \\ 1 & \text { if } x_{i} \geq x_{t}\end{cases}
$$

In this equation, $x_{t}$ represents the threshold which is often selected as the mean value of the time-series (X.-S. Zhang, Roy, \& Jensen, 2001).

- Calculate the complexity counter $c(N)$ representing the total number of distinct patterns/characters contained in the encoded binary string. This value is approaching an ultimate value $b(N)$ when the length of the sequence $N$ approaches to infinity.

$$
\begin{gathered}
c(N)=O(b(N)) \\
b(N)=\frac{N}{\log _{2} N}
\end{gathered}
$$

- Calculate the Kolmogorov complexity according to the rule described below,

$$
K L=\frac{c(N)}{b(N)}=c(N) \frac{\log _{2} N}{N}
$$

The $K L$ represents the quantity of information contained in the encoded timeseries. For cases where the length of the time-series is large enough, this value approaches 0 for periodic or regular time-series, and 1 for fully random timeseries (Mihailović, Mimić, Drešković, \& Arsenić, 2015).

\subsection{Preparation of the Kolmogorov complexity spectrum}

According to Mihailović, Mimić, Drešković, \& Arsenić (2015), the KL measure cannot differentiate between time-series with different amplitude variations and similar randomness trends. Moreover, the procedure in establishing the threshold for the KL measure may cause information losses regarding the structure of the time-series. To eliminate these drawbacks, Mihailović, Mimić, Nikolić-Djorić, \& Arsenić (2015) proposed a novel methodology which can be used to explore highly enhanced stochastic components of a time series by analysing Lempel-Ziv complexity of a range of amplitudes: which is called as "Kolmogorov spectra of complexity". The approach is as follows.

- Convert the time series into a sequence consisting of the characters that lay in the interval $[0,1]$ based on the rule described below.

$$
x_{i}=\frac{\left(X_{i}-X_{\min }\right)}{\left(X_{\max }-X_{\min }\right)}
$$

where $X$ is a time series obtained by a measuring process or as an output from a simulation model, $X_{i}$ is the $i^{\text {th }}$ value in $X, X_{\max }=\max (X)$ and $X_{\min }=\min (X)$. 
- Convert the normalised time series into a set of binary sequences $S_{i}^{k}$, $i=$ $1,2,3 \ldots, N, k=1,2,3 \ldots, N$, by comparing them with a series of thresholds $\left(x_{t, k}\right), k=$ $1,2,3 \ldots, N$, where each threshold element is equal to the corresponding element in the considered time series $\left(x_{i}\right), i=1,2,3 \ldots, N$.

$$
S_{i}^{k}= \begin{cases}0 & \text { if } x_{i}<x_{t, k} \\ 1 & \text { if } x_{i} \geq x_{t, k}\end{cases}
$$

- Apply LZA on each element of 0-1 sequences $\left(S_{i}^{k}\right)$ to obtain Kolmogorov complexity spectrum $\left(c_{i}\right), i=1,2,3 \ldots, N$. The Kolmogorov complexity spectrum enables the exploration of the time series over a range of amplitudes. Here, the maximum value of the Kolmogorov complexity spectrum is denoted as the Kolmogorov complexity spectrum highest value (KLM). KLM carries the information about the highest complexity among all complexities in the spectrum, and hence should be considered while analysing the randomness within system behaviours encrypted as a time-series.

\subsection{Measuring overall Kolmogorov complexity}

The overall Kolmogorov complexity (KLO) proposed by (Mihailović, Mimić, NikolićDjorić, \& Arsenić, 2015) offers a better understanding of complexity of dynamical systems, i.e. their time evolution and predictability. This measure is based on the Kolmogorov spectrum of complexity and can be calculated as follows:

$$
K L O=\frac{1}{N} \int_{X} K_{s}^{C} d x
$$

where, $K_{s}^{C}$ is the spectrum of Kolmogorov complexity, $d x$ is differential of the normalised amplitude, while $X$ is a domain of all normalised amplitudes over which this integral takes values (Mihailović, Mimić, Nikolić-Djorić, \& Arsenić, 2015). The complexity spectrum allows us to visualise complexity hidden in the coding rules of commonly used KL measure. In this sense, KLM and KLO can be considered as improved indicators, as KL only conveys average information about a time-series. This is also important, as KLO can differentiate between time-series with different amplitude variations and similar random components, thereby providing a distinction between different time series having close values of the KL and KLM measures. Thus, if available, the KLO can provide a better understanding of the Kolmogorov complexity of time-series.

\section{Case studies and results}

This section illustrates the implementation of the presented complexity measures using two DES models of discrete manufacturing systems derived from machining and assembly industries. 


\subsection{Case study one}

A simple manufacturing system producing one product has been implemented in a discrete event simulation model, for demonstrating the presented Kolmogorov complexity measures. The system configuration consists of six machines, each of which can only process one product at a time. The manufacturing system is illustrated in Figure 2. The system is considered balanced and the dispatching rule first-in-first-out is used for the selection of the product order to be performed by the workstations. The performance indicator chosen is the average product flow time, which is the average value of the differences between the completion (end) time and the arrival time of jobs processed in a particular time unit. The process cycle time of machines and product arrival rate are deterministic and kept constant in all simulations.

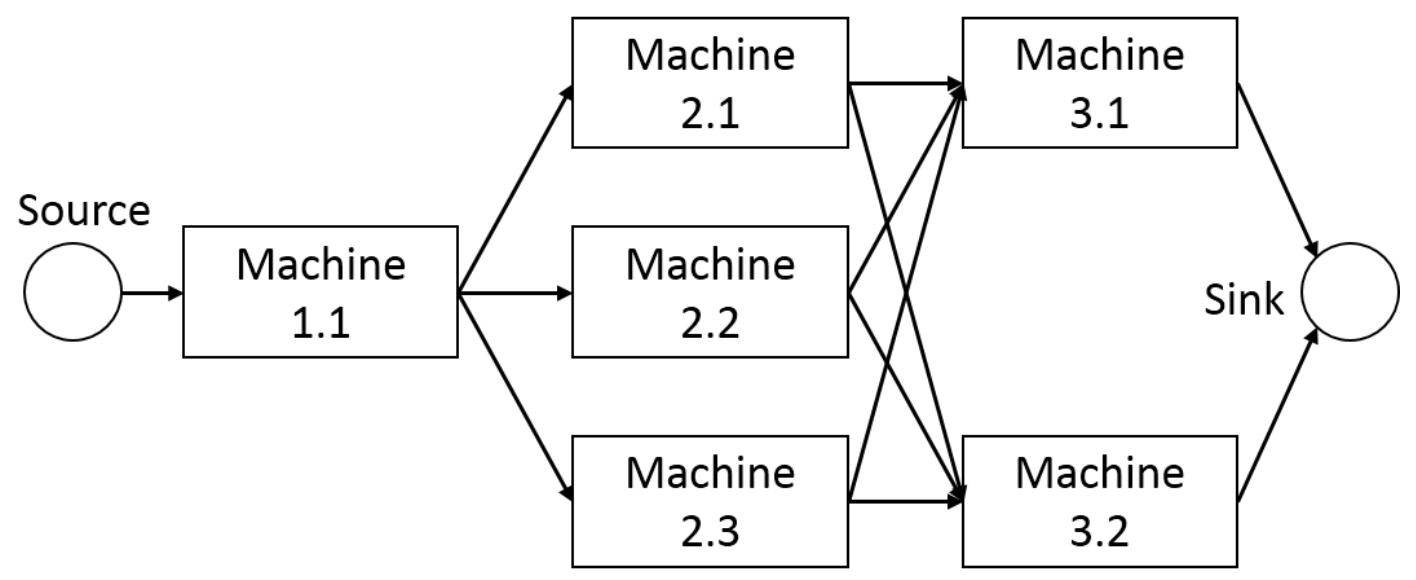

Figure 2. Example manufacturing system configuration.

It is often argued that the more complex a system is, the more it will cost to develop and operate and the less reliable it will be (Alkan et al., 2018). Based on this viewpoint, the performance of the Kolmogorov complexity measure is studied in the above-mentioned simulation model with varying machine reliabilities. Accordingly, the negative exponential distribution model with a mean denoted as $\lambda$ is assigned for mean time between failure (MTBF), and the normal distribution model with mean $\mu$ and standard deviation $\sigma^{2}$ is employed for the meantime to repair (MTTR) of system resources. Five scenarios with varying MTBF and MTTR values, which are given in Table 1, are investigated. Each simulation simulates 4000 units of time with average mean flow time recorded for each time unit. For each simulation, the inter-arrival time for the product is kept constant at five-time units.

Figure 3 shows the average mean flow time series sampled per time unit for each scenario. Operational complexity is calculated based on each of the three Kolmogorov complexity measures and is given in Table 1. The results are found to be in line with the previous hypothesis indicating an inverse relationship between the complexity and reliability of engineering systems. Accordingly, a decrease in system reliability is found to be accompanied by an increase in operational complexity for all three measures. This is reasonable, as systems become operationally unpredictable as the stochasticity involved in their operations increase, and this leads to a greater diversity within the consequent KPI time series which is reflected in higher Kolmogorov complexity values. Figure 4 illustrates Kolmogorov complexity spectra for individual production scenarios. Note that, in each case, the shape of the Kolmogorov complexity spectrum 
is qualitatively similar (although they differ in amplitude). This clearly indicates the presence of varying process stochasticity induced by the system reliability. KLO, is, therefore found to be very useful in detecting the impact of stochasticity on KPI time series.
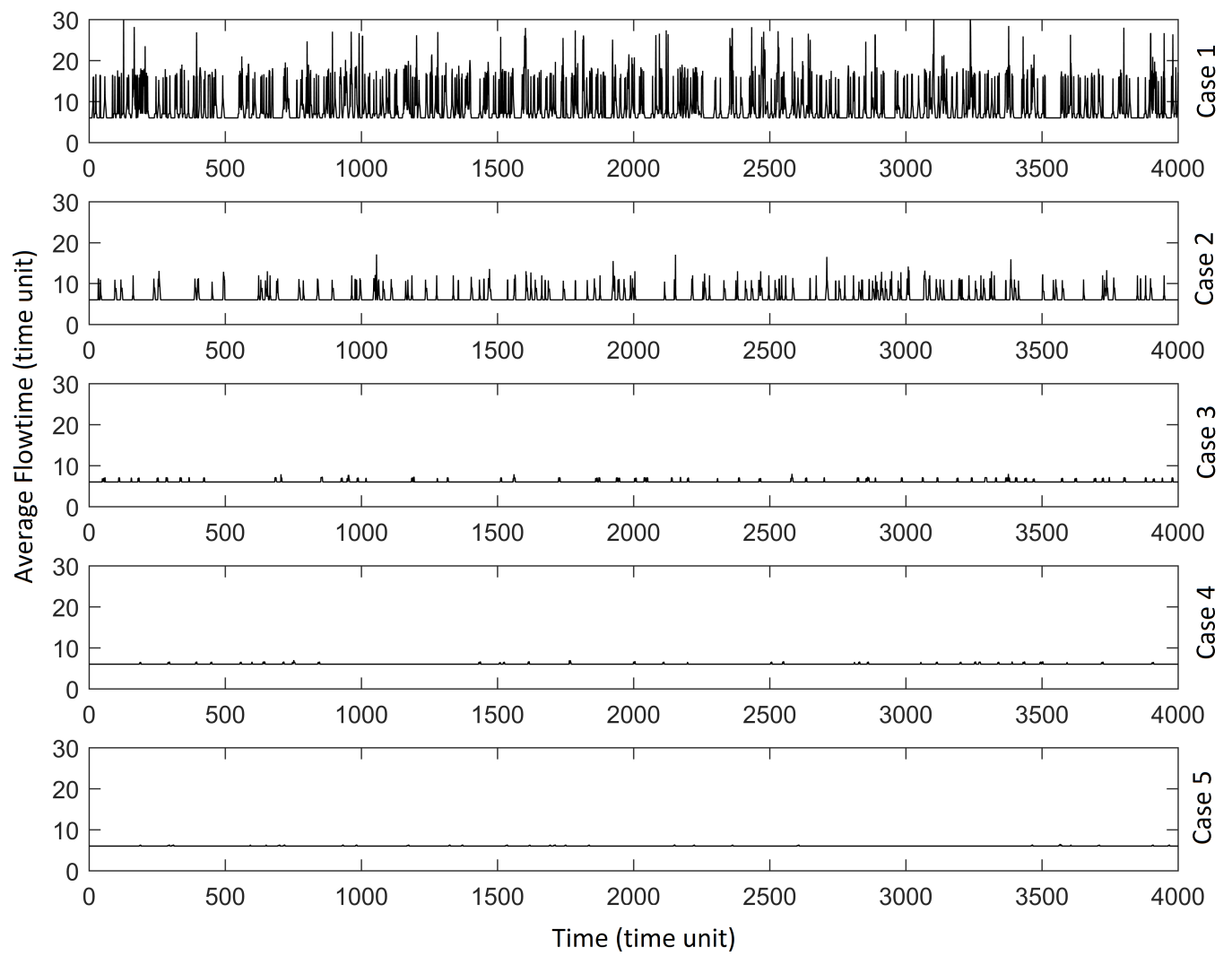

Figure 3. The average product flow time time-series for individual production scenarious with varying machine reliability.

Table 1. Operational complexity results of systems with varying machine reliability.

\begin{tabular}{lllllll}
\hline & \multicolumn{2}{l}{ MTBF } & \multicolumn{2}{c}{ MTTR } & \multicolumn{3}{c}{ Complexity results } \\
\cline { 2 - 7 } Case & $\lambda$ & $\mu$ & $\sigma^{2}$ & KL & KLM & KLO \\
\hline 1 & 50 & 10 & 0.5 & 0.6580 & 0.6610 & 0.2895 \\
2 & 150 & 5 & 0.25 & 0.3170 & 0.3260 & 0.1509 \\
3 & 300 & 1 & 0.1 & 0.2153 & 0.2183 & 0.1139 \\
4 & 500 & 0.5 & 0.05 & 0.1286 & 0.1286 & 0.0727 \\
5 & 1000 & 0.25 & 0.0125 & 0.1017 & 0.1017 & 0.0555 \\
\hline
\end{tabular}

\subsection{Case study two}

In this section, the presented algorithmic complexity measures are demonstrated on an industrial case study derived from a mixed model assembly line. The case study was originally designed in SimEvent/MATLAB to demonstrate the capabilities of SimEvent in analysing the impact of job scheduling on throughput. 


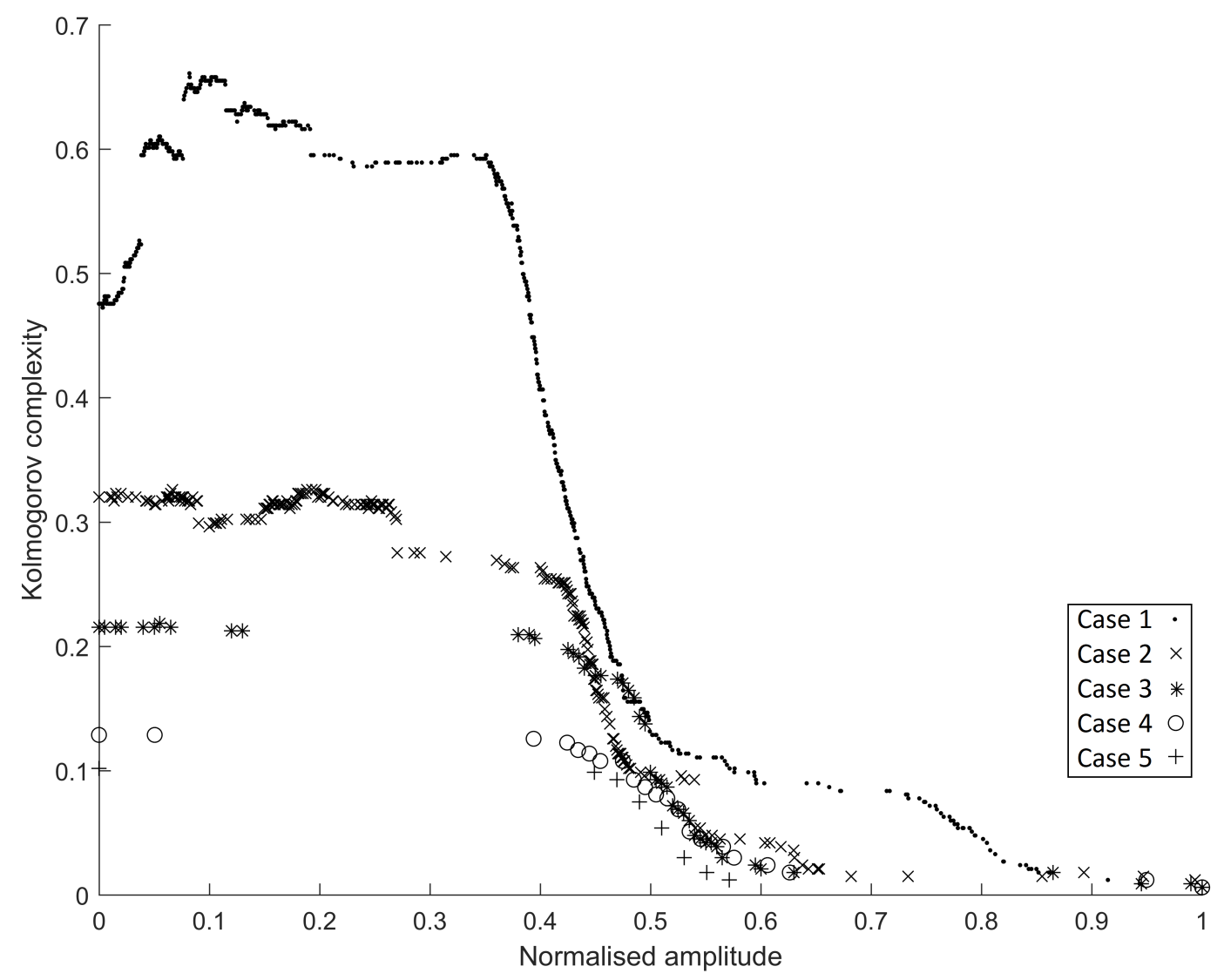

Figure 4. Kolmogorov complexity spectrum $c_{1}$ of product flowtime time series $x_{1}$ obtained through the discrete event simulation model. 


\subsubsection{Description of the plant}

The assembly line (Figure 5) can produce up to forty product variants; each requiring two parts (Part A and Part B) that correspond to that particular variant. To manufacture a particular variant, parts corresponding to the variant are brought together in the manufacturing area, where Part A goes through a specific blanking operation, and Part B goes through a specific milling operation. Both parts are then fastened, and the combined product goes through a finishing operation. Milling and fastening operations require human workers, whereas the finishing operation is performed by a robotic station. Human workers are responsible for loading and unloading products from two milling machines and one fastening machine. The finished products then enter the inspection area, where the finished product is certified to be completed or rejected and scrapped. The rejection rate is assumed to be $5 \%$ in the inspection area for all cases. Human workers are responsible for loading and unloading products from three inspection machines. The assembly line is considered to be balanced and operates on a $24 \mathrm{~h}$ basis with 3 repeated shifts.

\subsubsection{Description of experiments}

Operational complexity of the assembly line is studied using statistical design of computer experiments. The goal here is to maximize the knowledge regarding the causeeffect relationships between complexity drivers and operational complexity. The performance of the assembly line is tracked based on the average queue length of the buffer located between manufacturing and inspection areas. The average queue length of this specific buffer is selected as a performance indicator since any operational disturbance in the manufacturing area or the inspection area would lead to irregularities in the pattern of the time spent in the inspection buffer. This is also an appropriate indicator of whether two sub-systems, i.e. manufacturing and inspection, are working in a harmony. The assembly line is simulated in the MATLAB environment and analysed under various operational scenarios. For each scenario, the average queue length time series is tracked and used in the operational complexity calculations without any signal filtering. In the simulations, three human workers are employed for both manufacturing and inspection areas. The rejection rate during inspection is assumed to be $5 \%$ and kept constant for each scenario. The dispatching rule first-in-first-out is employed for processing the orders. A simulation runs for 2,000,000 time units, and average queue length KPI is sampled every 250-time units. The ratio of demand to maximum throughput per time unit is selected as 0.85 as is recommended by Efthymiou et al. (2014) and kept constant for every scenario.

\subsubsection{Effects of the job scheduling}

In this section, the operational complexity of the assembly line is investigated in the context of different job scheduling schemes. Five job scheduling schemes are employed to organise 20 weeks of production processed in weekly batches. In each batch, 200 product orders belonging to 40 different variants with a uniform random distribution are scheduled based on the following schemes:

- Schedule 1: Shortest job first on the blanking machine: This schedule prioritises the operations with the shortest cycle time on the blanking machine first and the longest ones at the end.

- Schedule 2: Shortest job first on the Milling machines: This schedule prioritises 


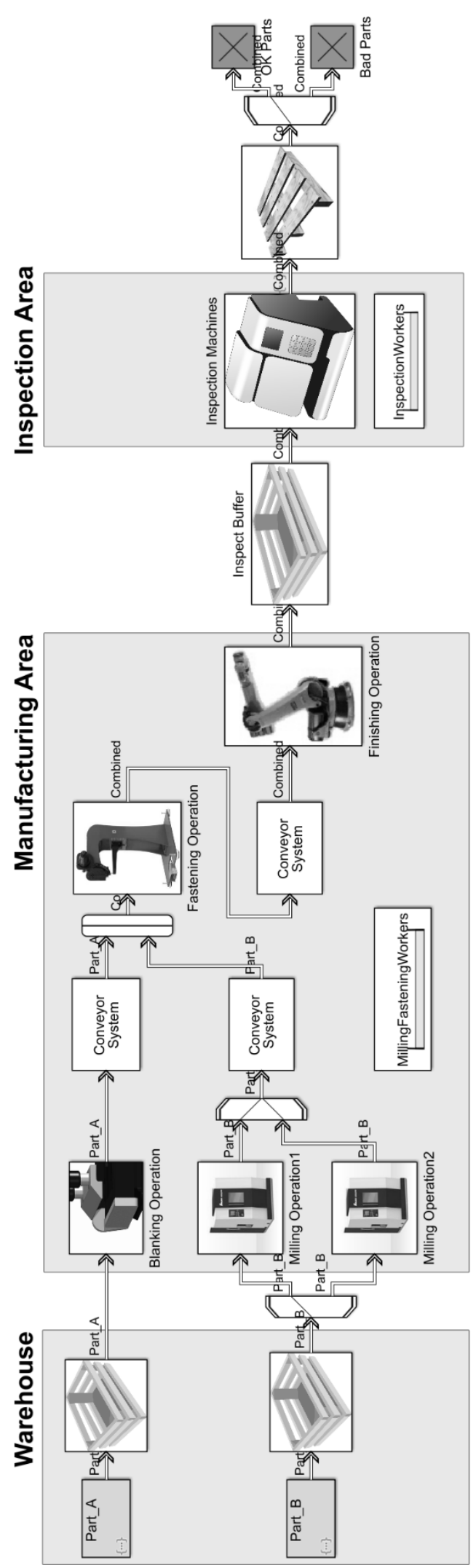

Figure 5. SimEvent model of the assembly line. 
operations based on their milling cycle times from shortest to longest.

- Schedule 3: Shortest job first on the Fastening machine: This schedule is designed based on the product fastening time; shortest fastening cycle time first and the longest ones at the end.

- Schedule 4: Shortest job first using the cumulative manufacturing time: This schedule prioritises product orders based on their cumulative cycle time on all the machines. The operations having the shortest cumulative cycle time is, therefore, put first and the longest ones put to the end.

- Schedules 5: Random schedule: This schedule is generated using a random permutation of the set of jobs.

Computer simulations are carried out using MATLAB SimEvents software with 5 replications for each of the five scenarios resulting in a total of 25 simulation experiments. In each simulation, average queue length KPI time-series of the inspection buffer was recorded as 8000 data points with a warm-up period of 250 discarded points. In order to isolate the effect of scheduling on the selected KPI, machine cycle times are assumed to be deterministic. Figure $\mathbf{6}$ illustrates the operational complexity of the assembly line for each job scheduling scheme. Table $\mathbf{2}$ displays complexity scores for individual production scenarios. It should be noted that Kolmogorov complexity measures are expected to be to zero if the system behaviours can be easily predicted, whereas, unpredictability/randomness is associated with higher complexity scores. According to the results, operational complexity of the assembly line is found to be below 0.1 for all cases indicating that the system is deterministic with very low complexity and very high predictability. Nevertheless, operational complexity of the system is found to be affected by the employed scheduling policies. Schedule 5 (i.e. random job ordering) produced the highest operational complexity (KLO $=0.0396$, $\mathrm{KL}=0.0595$, and $\mathrm{KLM}=0.0926$ ). This is reasonable, as schedule 5 follows a random product order, whereas, other schedules execute operations by prioritising particular variants. It is interesting to note that, schedules 1 and 4 have displayed the lowest operational complexity with the same Kolmogorov complexity value $(\mathrm{KL}=0.0265)$. This indicates the presence of a similar degree of random components in their performance time-series. The KLO measure, however, distinguished between the complexity of the two schedules as can be seen in Figure 7. This additional information is not contained in KL and KLM measures and allows us to conclude that schedule $1(\mathrm{KLO}=0.0275)$ has larger variability of amplitudes and produced more operational complexity than schedule $4(\mathrm{KLO}=0.0261)$ if the whole spectrum of Kolmogorov complexity is taken into account. This is also reasonable as schedule 4 provides a more holistic approach in job scheduling; thereby minimising the impacts of operational uncertainties in the long run to a greater extent than approaches prioritising particular aspects/areas of a manufacturing system. It is interesting to note that, schedules 2 (KLO $=0.0280$, $\mathrm{KL}=0.0398$, and $\mathrm{KLM}=0.0763)$ and $3(\mathrm{KLO}=0.0290, \mathrm{KL}=0.0364$, and KLM $=0.0596)$ have produced relatively high operational complexity by comparison with both schedules 1 and 4 based on KL and KLO measures.

\subsubsection{Effects of process stochasticity}

This section investigates the relationships between process stochasticity and operational complexity using the KL, KLM and KLO algorithmic complexity measures for data from discrete event simulations. Towards this aim, 8 computer experiments with 5 replications each producing 40 KPI time-series were analysed. Stochasticity is only 


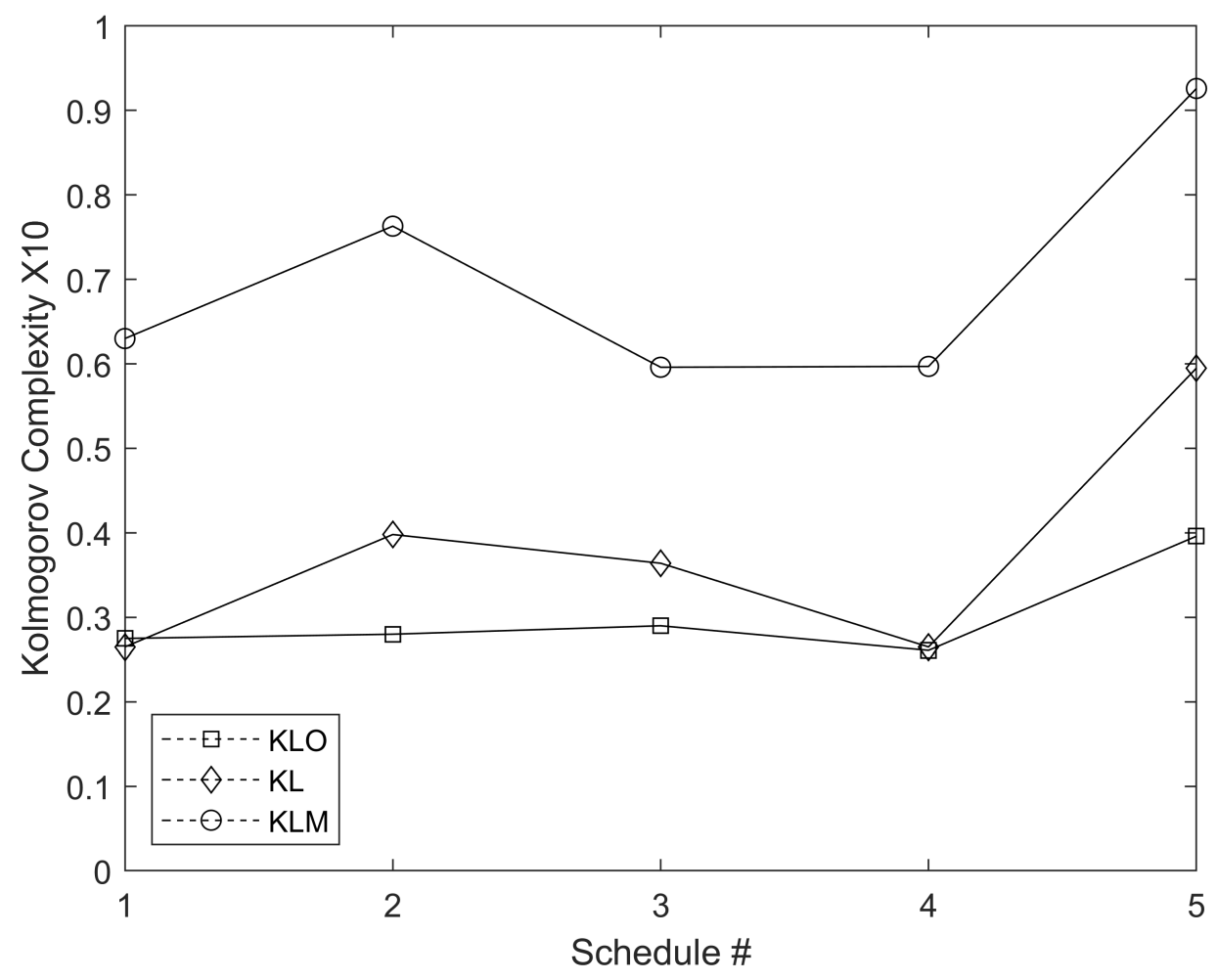

Figure 6. Three measures of operational complexity for each of five job scheduling schemes.

Table 2. Operational complexity results of systems under different job schedules.

\begin{tabular}{llll}
\hline & KLO & KL & KLM \\
\hline Schedule 1 & 0.0275 & 0.0265 & 0.0630 \\
Schedule 2 & 0.0280 & 0.0398 & 0.0763 \\
Schedule 3 & 0.0290 & 0.0364 & 0.0596 \\
Schedule 4 & 0.0261 & 0.0265 & 0.0597 \\
Schedule 5 & 0.0396 & 0.0595 & 0.0926 \\
\hline
\end{tabular}



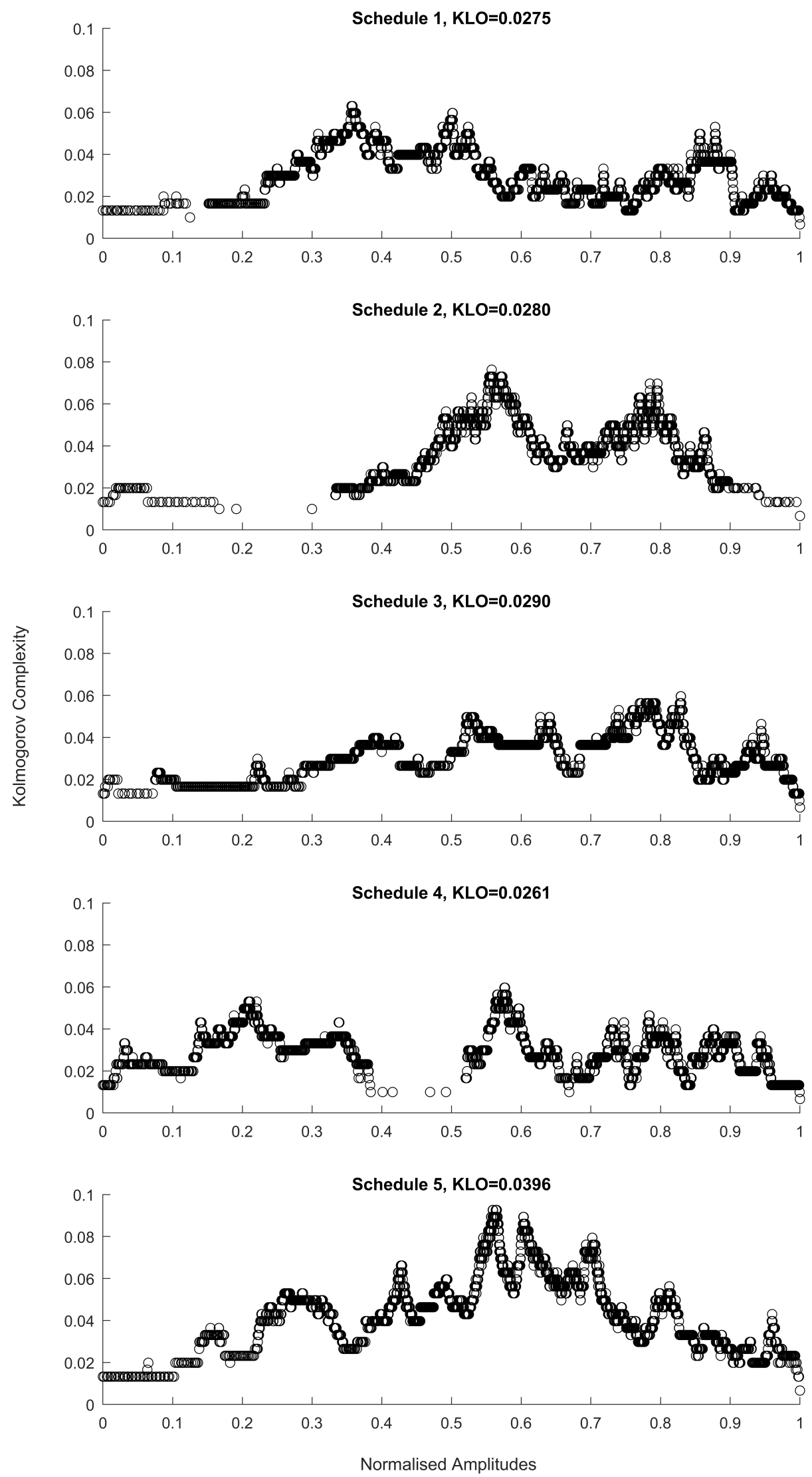

Figure 7. Kolmogorov complexity spectrum $c_{1}$ of average queue length time series $x_{1}$ obtained through the SimEvent model. 
introduced to the cycle time of system resources, where the variation in operation completion times are assumed as 2.5\%, 5\%, 7.5\%, 10\%, 12.5\%, 15\%, 17.5\% and $20 \%$. The warm-up time and run time of simulations are kept constant and the same as the experiments presented in the previous section. Only one product variant (Variant 1) was fed to the system to better analyse the effects of stochasticity in isolation.

Figure 8 shows the relationship between process stochasticity and operational complexity. As expected, a positive correlation is found between operational complexity and process stochasticity defined by process cycle-time variations. Here, we use a linear fit to describe the relationship trend. However, non-linear models could be used to more accurately define the correct relationship trend.

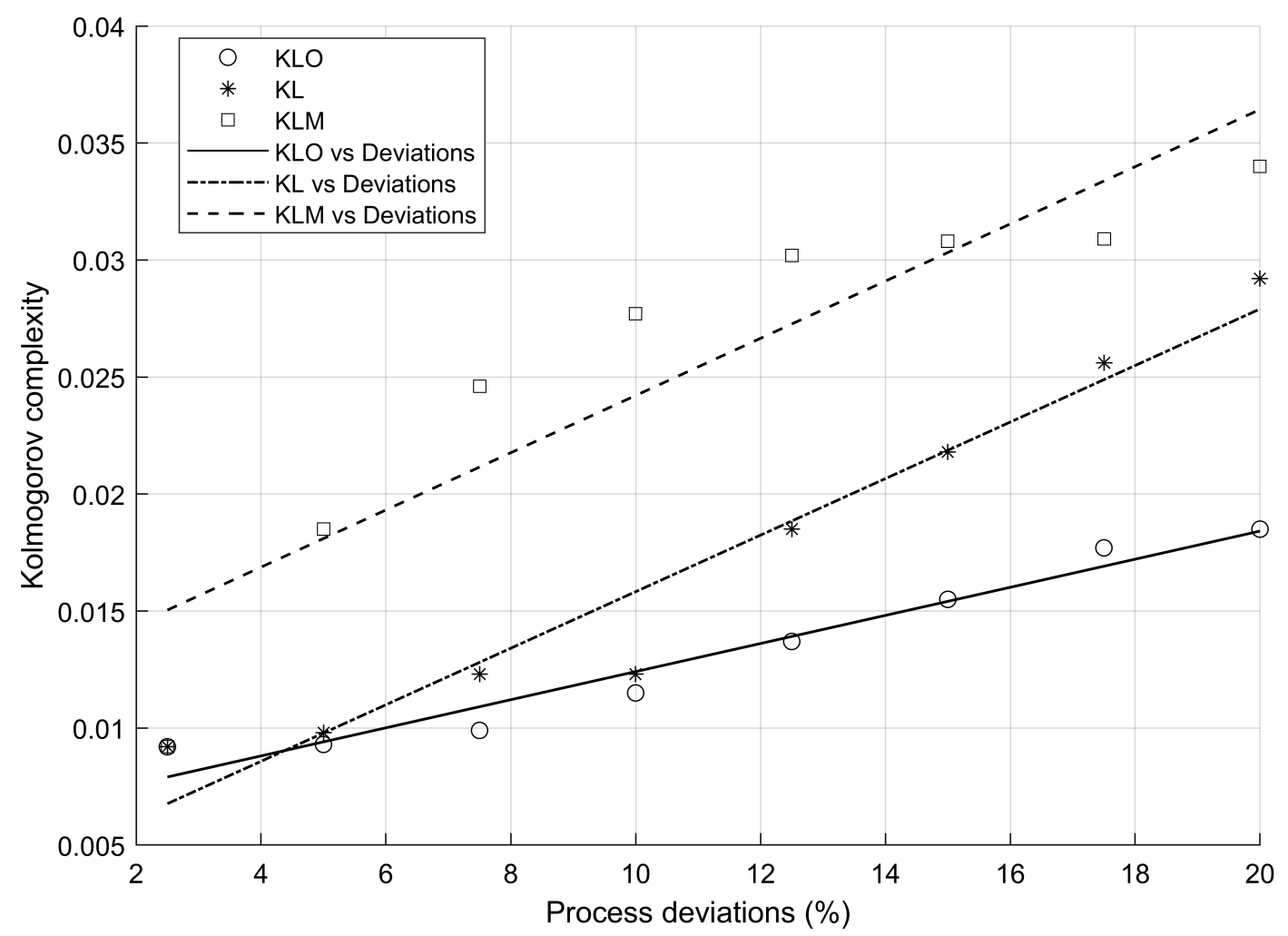

Figure 8. The relationship between Kolmogorov complexity and process stochasticity.

\subsubsection{Effects of the product mix ratio}

Increased product variety is one of the main factors affecting operational complexity of manufacturing systems (S. N. Samy \& ElMaraghy, 2010). Handling increased product variety necessitates the manufacturing system to quickly react and adapt to manufacturing disturbances. Poor variety management can result in stochastic line balancing problems (Alkan et al., 2018). In this section, the relationship between product variety and operational complexity is studied using a series of computer experiments. To simplify the experiment, only product variants 1 and 2 are considered. In these experiments, the effects of product variety are analysed based on five levels of product mix ratios, i.e. 50-50\%, 60-40\%, 70-30\%, 80-20\%, 90-10\%. The correlation between operational complexity and product mix ratios is developed using trend analysis. Similar to study carried out in (Efthymiou et al., 2014), an information-theoretic approach is 
used to characterise the effect of product variety. The entropy is computed based on the percentage of each variant in the product mix. Accordingly, information entropy $H$ induced by the product varieties is calculated as follows:

$$
H=\sum_{i=1}^{2}-p_{i} \log _{2} p_{i}
$$

where $p_{i}$ represents the percentage of the product in the product mix.

Table 3 shows KLO, KL, KLM and $\mathrm{H}$ values for each product mix case. Interestingly, KL measure was unable to differentiate operational complexity of the manufacturing system processing two product variants with mix ratios of $60-40 \%, 70-30 \%$, $80-20 \%$ and $90-10 \%$. The authors believe that this may be associated with the length of the selected KPI time-series, as a larger number of observations may be required to distinguish time series with similar trends. On the other hand, KLO and KLM measures delivered results with better resolution for the selected time-series length, thereby providing an alternative indicator where KL measure is incapable to compare complexity of time-series. Moreover, as opposed to Efthymiou et al. (2014) where the relationship between Kolmogorov complexity and product mix entropy is explained with a linear fit, an exponential fit was found to be better suited for the relationships between KLO and KLM measures and product mix entropy (Figure 9). Accordingly, the R-squared values are found as 0.9940 .999 for the relationships between KLO-H and KLM-H, respectively.

Table 3. Operational complexity results of the system performing under varying product mix ratios.

\begin{tabular}{llllll}
\hline Variant 1 & Variant 2 & $\mathrm{H}$ & $\mathrm{KLO}$ & $\mathrm{KL}$ & $\mathrm{KLM}$ \\
\hline $50 \%$ & $50 \%$ & 1.00000 & 0.0166 & 0.0263 & 0.0823 \\
$60 \%$ & $40 \%$ & 0.97095 & 0.0151 & 0.0198 & 0.0758 \\
$70 \%$ & $30 \%$ & 0.88129 & 0.0137 & 0.0198 & 0.0626 \\
$80 \%$ & $20 \%$ & 0.72193 & 0.0129 & 0.0198 & 0.0494 \\
$90 \%$ & $10 \%$ & 0.46900 & 0.0123 & 0.0198 & 0.0428 \\
\hline
\end{tabular}

\section{Discussion}

This research presents an operational complexity quantification method based on the application of three complementary Kolmogorov complexity measures on univariate production KPI time-series recorded sequentially over equal time increments. The article, for the first time, implements Kolmogorov complexity spectrum and Overall Kolmogorov complexity measures within the domain of manufacturing systems engineering. The proposed approach can be used in quantitatively assessing operational manufacturing systems complexity during both design and operational life-cycle phases. The approach objectively links operational complexity to production system KPIs, thereby allowing designers/managers to better understand the cause-effect relations between the factors generating complexity and their implications on operational disruptions. This way, the approach supports operational shop-floor decision-making activities.

In the manufacturing domain, the proposed approach has clear advantages over the previously presented complexity measures. First, the approach involves the combined 

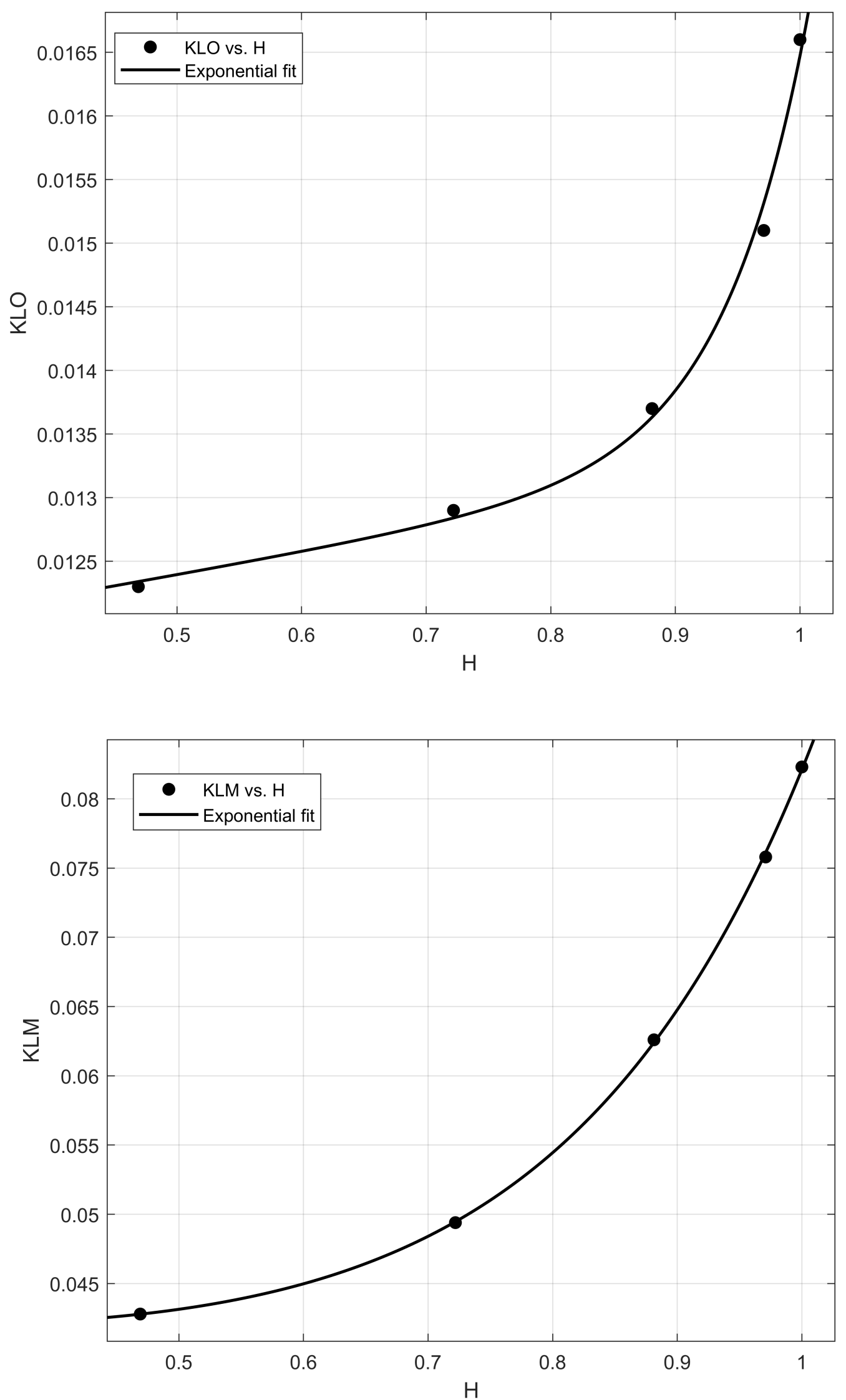

19

Figure 9. The exponential impact of product $\operatorname{mix}$ on the operational complexity $\left(R_{K L O}^{2}=0.994\right.$, $\left.R_{K L M}^{2}=0.999\right)$. 
used of three Kolmogorov complexity measures, resulting in a better complexity assessment resolution, which can be especially useful in comparing alternate manufacturing system designs that generate KPI time series with different amplitude variations and similar random components. Kolmogorov complexity measures do not require setting in time-series onto a high dimensional representation, which is often needed in measures derived from chaos and non-linear dynamics theories such as bifurcation diagrams and Maximal Lyapunov Exponent Testing (Efthymiou et al., 2016). Furthermore, Kolmogorov complexity measures are easy to use and apply as they can be readily calculated for any type of time-series and are constrained by assumptions regarding the probability law of the process generating the time series (Mihailović, Mimić, NikolićDjorić, \& Arsenić, 2015). The approach presented here is also a data-driven method and can be easily embraced by existing manufacturing control and advanced manufacturing decision-support systems within the broader framework of Industry 4.0. The approach can be embedded within the existing IoT-enabled data analytics and visualisation platforms to provide more flexibility in decision-making processes. In such a way, operational complexity can be used as a new decision-making criterion alongside the existing ones such as: cost, time, manufacturing flexibility, etc.

Along with its advantages, the presented approach has a set of drawbacks that need to be addressed before employing it as an industry-wide practice. Firstly, Kolmogorov complexity measures are highly sensitive to the length of time-series and the noise which occurs during the observation process. To overcome this, KPI time-series obtained through on-site measurements should be subjected to a pre-screening phase where the quality of the data is checked and verified. The calculation of Kolmogorov complexity and interpretation of complexity results may require expertise, and hence personnel training should be considered in order to make such approaches effective and reliable. Although the approach provides an objective complexity calculation solely based on the irregularities hidden in KPI time-series, the selection of the KPI timeseries to be investigated is subjective and requires expertise. Moreover, the approach assesses operational complexity of a manufacturing system based on a univariate KPI time-series consisting of the observations of a single variable. However, manufacturing systems are highly complex socio-technical systems depicting behaviours across multiple dimensions. Therefore, the proposed approach should be extended to have the capability to analyse multivariate KPI time-series consisting of multiple KPIs. Kolmogorov complexity evaluation based on multivariate time-series is expected to provide a more detailed picture of the uncertainty associated with the manufacturing system operations being considered.

Table 4 summarises the advantages and disadvantages of the proposed approach and previous operational complexity quantification approaches within the domain of manufacturing systems engineering.

\section{Conclusion and future works}

In this article, three complementary algorithmic complexity measures, i.e. Kolmogorov complexity, Kolmogorov complexity spectrum highest value and overall Kolmogorov complexity, are presented to assess the operational complexity of manufacturing systems which is believed to be hidden in systems' KPI time-series. The presented measures enable an objective way to compare system/process designs, and can be used in selecting optimal system parameters with regard to maximising the predictability of manufacturing operations. The presented measures are demonstrated using an 
Table 4. A comparison between the proposed approach and other operational complexity measures.

\begin{tabular}{|c|c|c|}
\hline Approach & Strengths & Weaknesses \\
\hline $\begin{array}{l}\text { Information entropy } \\
\text { based methods }\end{array}$ & Objective & $\begin{array}{l}\text { Inter-dependency assumption } \\
\text { Subjective state definitions } \\
\text { Probability estimation accuracy } \\
\text { Expertise requirements }\end{array}$ \\
\hline Chaos theory and & Objective & Large data requirements \\
\hline non-linear dynamics & $\begin{array}{l}\text { Links complexity to KPIs } \\
\text { Captures the impact of change }\end{array}$ & $\begin{array}{l}\text { Sensitivity to noise } \\
\text { Sensitivity to sample size } \\
\text { Expertise requirements } \\
\text { Limited in design phases }\end{array}$ \\
\hline $\begin{array}{l}\text { Surveys based } \\
\text { methods }\end{array}$ & $\begin{array}{l}\text { Early deployment is possible } \\
\text { Captures human perceptions }\end{array}$ & $\begin{array}{l}\text { Time consuming } \\
\text { Subjective } \\
\text { Not applicable in design phases }\end{array}$ \\
\hline $\begin{array}{l}\text { Heuristics based } \\
\text { methods }\end{array}$ & $\begin{array}{l}\text { Industry friendly } \\
\text { Quick }\end{array}$ & $\begin{array}{l}\text { Ad-hoc methods } \\
\text { Subjective } \\
\text { Accuracy problems }\end{array}$ \\
\hline $\begin{array}{l}\text { Kolmogorov complexity } \\
\text { measures }\end{array}$ & $\begin{array}{l}\text { Objective } \\
\text { Links complexity to KPIs } \\
\text { Captures the impact of change }\end{array}$ & $\begin{array}{l}\text { Data requirements } \\
\text { Sensitivity to noise } \\
\text { Sensitivity to sample size } \\
\text { Subjectivity in selecting KPIs } \\
\text { Expertise requirements }\end{array}$ \\
\hline
\end{tabular}

industrial case study derived from a mixed model assembly line; operational complexity is investigated with respect to varying job schedules, process deviations and product-mix ratios. The results showed that the presented measures can be used within a real-time process optimisation context, where manufacturing disturbance handling can be achieved through prioritising the predictability of manufacturing processes. This will ultimately lead to better productivity by reducing uncertainty involved in manufacturing shop-floor decision-making activities.

It is envisioned that the following two developments of the approach could be made. First, the approach presented here will be extended to include the synchronized analyses of multivariate KPI time-series to provide a better picture of operational manufacturing systems complexity. A series of simulation experiments will be carried out to verify the sensitivity of the approach across time-series with various length and noise amplitudes. Moreover, the presented approach will be embedded within an Industry 4.0 based data-analytics and visualisation platform where multiple KPI-time series can be streamlined and analysed to assess operational predictability of manufacturing systems and the cause-effect relationships between complexity and performance.

\section{Disclosure statement}

No potential conflict of interest was reported by the authors.

\section{References}

Ahmad, M., Alkan, B., Ahmad, B., Vera, D., Harrison, R., Meredith, J., \& Bindel, A. (2016). The use of a complexity model to facilitate in the selection of a fuel cell assembly sequence. Procedia CIRP, 44, 169-174. 
Alkan, B. (2018). A complexity modelling approach to support early life-cycle phases of assembly automation systems (Unpublished doctoral dissertation). University of Warwick.

Alkan, B. (2019). An experimental investigation on the relationship between perceived assembly complexity and product design complexity. International Journal on Interactive Design and Manufacturing (IJIDeM), 13(3), 1145-1157.

Alkan, B., \& Harrison, R. (2019). A virtual engineering based approach to verify structural complexity of component-based automation systems in early design phase. Journal of Manufacturing Systems, 53, 18-31.

Alkan, B., Vera, D., Ahmad, B., \& Harrison, R. (2017). A method to assess assembly complexity of industrial products in early design phase. IEEE Access, 6, 989-999.

Alkan, B., Vera, D., Ahmad, M., Ahmad, B., \& Harrison, R. (2016a). Design evaluation of automated manufacturing processes based on complexity of control logic. Procedia CIRP, $50,141-146$.

Alkan, B., Vera, D., Ahmad, M., Ahmad, B., \& Harrison, R. (2016b). A model for complexity assessment in manual assembly operations through predetermined motion time systems. Procedia Cirp, 44, 429-434.

Alkan, B., Vera, D., Chinnathai, M. K., \& Harrison, R. (2017). Assessing complexity of component-based control architectures used in modular automation systems. International Journal of Computer and Electrical Engineering, 9(1), 393-402.

Alkan, B., Vera, D. A., Ahmad, M., Ahmad, B., \& Harrison, R. (2018). Complexity in manufacturing systems and its measures: a literature review. European Journal of Industrial Engineering, 12(1), 116-150.

Amrina, E., \& Vilsi, A. L. (2015). Key performance indicators for sustainable manufacturing evaluation in cement industry. Procedia Cirp, 26(1), 19-23.

Assad, F., Alkan, B., Chinnathai, M., Ahmad, M., Rushforth, E., \& Harrison, R. (2019). A framework to predict energy related key performance indicators of manufacturing systems at early design phase. Procedia CIRP, $81,145-150$

Brockwell, P. J., \& Davis, R. A. (2016). Introduction to time series and forecasting. springer.

Calinescu, A., Efstathiou, J., Schirn, J., \& Bermejo, J. (1998). Applying and assessing two methods for measuring complexity in manufacturing. Journal of the Operational Research Society, 49(7), 723-733.

Chryssolouris, G. (2013). Manufacturing systems: theory and practice. Springer Science \& Business Media.

Chryssolouris, G., Efthymiou, K., Papakostas, N., Mourtzis, D., \& Pagoropoulos, A. (2013). Flexibility and complexity: is it a trade-off? International Journal of Production Research, 51(23-24), 6788-6802.

Chryssolouris, G., Giannelos, N., Papakostas, N., \& Mourtzis, D. (2004). Chaos theory in production scheduling. CIRP Annals, 53(1), 381-383.

Cover, T. M., \& Thomas, J. A. (2012). Elements of information theory. John Wiley \& Sons.

Deb, C., Zhang, F., Yang, J., Lee, S. E., \& Shah, K. W. (2017). A review on time series forecasting techniques for building energy consumption. Renewable and Sustainable Energy Reviews, 74, 902-924.

Deshmukh, A. V., Talavage, J. J., \& Barash, M. M. (1998). Complexity in manufacturing systems, part 1: Analysis of static complexity. IIE transactions, 30(7), 645-655.

Donner, R., Scholz-Reiter, B., \& Hinrichs, U. (2008). Nonlinear characterization of the performance of production and logistics networks. Journal of Manufacturing Systems, 27(2), $84-99$.

Efstathiou, J., Calinescu, A., \& Blackburn, G. (2002). A web-based expert system to assess the complexity of manufacturing organizations. Robotics and Computer-Integrated Manufacturing, 18(3-4), 305-311.

Efthymiou, K. (2013). On the assessment of manufacturing systems complexity. University of Patras.

Efthymiou, K., Mourtzis, D., Pagoropoulos, A., Papakostas, N., \& Chryssolouris, G. (2016). Manufacturing systems complexity analysis methods review. International Journal of Com- 
puter Integrated Manufacturing, 29(9), 1025-1044.

Efthymiou, K., Pagoropoulos, A., Papakostas, N., Mourtzis, D., \& Chryssolouris, G. (2012). Manufacturing systems complexity review: challenges and outlook. Procedia CIRP, 3, 644649.

Efthymiou, K., Pagoropoulos, A., Papakostas, N., Mourtzis, D., \& Chryssolouris, G. (2014). Manufacturing systems complexity: An assessment of manufacturing performance indicators unpredictability. CIRP Journal of Manufacturing Science and Technology, 7(4), 324-334.

ElMaraghy, H., Schuh, G., ElMaraghy, W., Piller, F., Schönsleben, P., Tseng, M., \& Bernard, A. (2013). Product variety management. Cirp Annals, 62(2), 629-652.

ElMaraghy, H. A. (2005). Flexible and reconfigurable manufacturing systems paradigms. International journal of flexible manufacturing systems, 17(4), 261-276.

ElMaraghy, W., \& Urbanic, R. J. (2003). Modelling of manufacturing systems complexity. CIRP Annals, 52(1), 363-366.

Falck, A.-C., Örtengren, R., \& Rosenqvist, M. (2012). Relationship between complexity in manual assembly work, ergonomics and assembly quality. In Ergonomics for sustainability and growth, nes 2012 (nordiska ergonomisällskapet) konferens, saltsjöbaden, stockholm, 1922 augusti, 2012.

Frizelle, G., \& Suhov, Y. M. (2001). An entropic measurement of queueing behaviour in a class of manufacturing operations. Proceedings of the Royal Society of London. Series A: Mathematical, Physical and Engineering Sciences, 457(2011), 1579-1601.

Frizelle, G., \& Woodcock, E. (1995). Measuring complexity as an aid to developing operational strategy. International Journal of Operations $\&$ Production Management.

Garbie, I. H., \& Shikdar, A. (2010). Design for manufacturing systems complexity: a perspective approach. In Asme 2010 10th biennial conference on engineering systems design and analysis (pp. 751-762).

Gell-Mann, M. (1995). The quark and the jaguar: Adventures in the simple and the complex. Macmillan.

Giannelos, N., Papakostas, N., Mourtzis, D., \& Chryssolouris, G. (2007). Dispatching policy for manufacturing jobs and time-delay plots. International Journal of Computer Integrated Manufacturing, 20(4), 329-337.

Ibáñez-Molina, A. J., Iglesias-Parro, S., Soriano, M. F., \& Aznarte, J. I. (2015). Multiscale lempel-ziv complexity for eeg measures. Clinical Neurophysiology, 126(3), 541-548.

Irani, Z. (2010). Investment evaluation within project management: an information systems perspective. Journal of the Operational Research Society, 61(6), 917-928.

Katzorke, I., \& Pikovsky, A. (2000). Chaos and complexity in a simple model of production dynamics. Discrete Dynamics in Nature and Society, 5(3), 179-187.

Kim, Y.-S. (1999). A system complexity approach for the integration of product development and production system design (Unpublished doctoral dissertation). Massachusetts Institute of Technology.

Kohr, D., Ahmad, M., Alkan, B., Chinnathai, M. K., Budde, L., Vera, D. A., ... Harrison, R. (2018). Proposing a holistic framework for the assessment and management of manufacturing complexity through data-centric and human-centric approaches. In Complexis (pp. 86-93).

Lempel, A., \& Ziv, J. (1976). On the complexity of finite sequences. IEEE Transactions on information theory, 22(1), 75-81.

Massotte, P. (1996). Behavioural analysis of a complex system. The International Journal of Advanced Manufacturing Technology, 12(1), 66-76.

Mattsson, S., Gullander, P., \& Davidsson, A. (2011). Method for measuring production complexity. In 28th international manufacturing conference.

Mattsson, S., Tarrar, M., \& Fast-Berglund, A. (2016). Perceived production complexityunderstanding more than parts of a system. International Journal of Production Research, 54(20), 6008-6016.

Mihailović, D. T., Mimić, G., Drešković, N., \& Arsenić, I. (2015). Kolmogorov complexity based information measures applied to the analysis of different river flow regimes. Entropy, 
$17(5), 2973-2987$.

Mihailović, D. T., Mimić, G., Nikolić-Djorić, E., \& Arsenić, I. (2015). Novel measures based on the kolmogorov complexity for use in complex system behavior studies and time series analysis. Open Physics, 13(1).

Montgomery, D. C., Jennings, C. L., \& Kulahci, M. (2015). Introduction to time series analysis and forecasting. John Wiley \& Sons.

Mourtzis, D., Doukas, M., \& Psarommatis, F. (2013). Design and operation of manufacturing networks for mass customisation. CIRP Annals, 62(1), 467-470.

Papakostas, N., Efthymiou, K., Mourtzis, D., \& Chryssolouris, G. (2009). Modelling the complexity of manufacturing systems using nonlinear dynamics approaches. CIRP annals, 58(1), 437-440.

Papakostas, N., \& Mourtzis, D. (2007). An approach for adaptability modeling in manufacturing-analysis using chaotic dynamics. CIRP annals, 56(1), 491-494.

Rivolta, M. W., Migliorini, M., Aktaruzzaman, M., Sassi, R., \& Bianchi, A. M. (2014). Effects of the series length on lempel-ziv complexity during sleep. In Engineering in medicine and biology society (embc), 2014 36th annual international conference of the ieee (pp. 693-696).

Samy, S., \& ElMaraghy, H. (2012). A model for measuring complexity of automated and hybrid assembly systems. The International Journal of Advanced Manufacturing Technology, 62(58), 813-833.

Samy, S. N., \& ElMaraghy, H. (2010). A model for measuring products assembly complexity. International Journal of Computer Integrated Manufacturing, 23(11), 1015-1027.

Sarkis, J. (1997). An empirical analysis of productivity and complexity for flexible manufacturing systems. International Journal of Production Economics, 48(1), 39-48.

Schmitz, J., Van Beek, D., \& Rooda, J. (2002). Chaos in discrete production systems? Journal of Manufacturing Systems, 21(3), 236-246.

Schoettl, F., Paefgen, M.-C., \& Lindemann, U. (2014). Approach for measuring change-induced complexity based on the production architecture. In 47 th cirp conference on manufacturing systems (pp. 934-939).

Scholz-Reiter, B., Freitag, M., \& Schmieder, A. (2002). Modelling and control of production systems based on nonlinear dynamics theory. CIRP Annals-Manufacturing Technology, $51(1), 375-378$.

Shahid, J. (2019). Influxdb documentation. Release.

Shannon, C. E. (2001). A mathematical theory of communication. ACM SIGMOBILE mobile computing and communications review, 5(1), 3-55.

Sivadasan, S., Efstathiou, J., Calinescu, A., \& Huatuco, L. H. (2006). Advances on measuring the operational complexity of supplier-customer systems. European Journal of Operational Research, 171 (1), 208-226.

Sivadasan, S., Smart, J., Huaccho Huatuco, L., \& Calinescu, A. (2010). Operational complexity and supplier-customer integration: case study insights and complexity rebound. Journal of the Operational Research Society, 61(12), 1709-1718.

Volz, J., \& Rabenstein, B. (2015). Prometheus: A next-generation monitoring system (workshop).

Vrabič, R., \& Butala, P. (2011). Computational mechanics approach to managing complexity in manufacturing systems. CIRP annals, 60(1), 503-506.

Wiendahl, H.-P., \& Scheffczyk, H. (1999). Simulation based analysis of complex production systems with methods of nonlinear dynamics. CIRP Annals, 48(1), 357-360.

Windt, K., Philipp, T., \& Böse, F. (2008). Complexity cube for the characterization of complex production systems. International Journal of Computer Integrated Manufacturing, 21(2), $195-200$.

Wu, Y., Frizelle, G., \& Efstathiou, J. (2007). A study on the cost of operational complexity in customer-supplier systems. International Journal of Production Economics, 106(1), 217229.

Wu, Y. R., Huatuco, L. H., Frizelle, G., \& Smart, J. (2013). A method for analysing operational complexity in supply chains. Journal of the Operational Research Society, 64 (5), 654-667. 
Yentes, J. M., Hunt, N., Schmid, K. K., Kaipust, J. P., McGrath, D., \& Stergiou, N. (2013). The appropriate use of approximate entropy and sample entropy with short data sets. Annals of biomedical engineering, 41(2), 349-365.

Zhang, T., \& Efstathiou, J. (2006). The complexity of mass customization systems under different inventory strategies. International Journal of Computer Integrated Manufacturing, $19(5), 423-433$.

Zhang, X.-S., Roy, R. J., \& Jensen, E. W. (2001). Eeg complexity as a measure of depth of anesthesia for patients. IEEE transactions on biomedical engineering, 48(12), 1424-1433.

Zhang, Y., Wei, S., Liu, H., Zhao, L., \& Liu, C. (2016). A novel encoding lempel-ziv complexity algorithm for quantifying the irregularity of physiological time series. Computer methods and programs in biomedicine, 133, 7-15.

Zhang, Z. (2012). Manufacturing complexity and its measurement based on entropy models. The International Journal of Advanced Manufacturing Technology, 62(9-12), 867-873. 